\title{
THE EXTERNAL AGGREGATION NEWTON'S METHOD FOR SOLVING NONLINEAR EQUATIONS AND APPLICATIONS
}

\author{
MARIJA PAUNOVIĆ ${ }^{1}$, DEJAN ĆEBIĆ ${ }^{2}$, NEBOJŠA RALEVIĆ ${ }^{3, \star}$ \\ ${ }^{1}$ Faculty of Hotel Management and Tourism in Vrnjacka Banja, University of Kragujevac, Vrnjacka Banja, Serbia \\ ${ }^{2}$ Faculty of Mining and Geology, University of Belgrade, Belgrade, Serbia \\ ${ }^{3}$ Faculty of Technical Sciences, University of Novi Sad, Novi Sad, Serbia
}

\begin{abstract}
Different modified Newton's methods are widely used in modern computational engineering science, applied mechanics, economics, optimization problems and other disciplines. On the other hand aggregation of information takes a significant place in many knowledge-based systems, where aggregation of data or values is needed. In this paper we theoretically analysed a new modification of Newton's method based on aggregation function applied on finding multiple roots of nonlinear equations, and numerically verified theoretical results on the examples with simple and multiple roots. Numerical analysis of the proposed approach with obtained results and the related discussion are presented on examples in practice.
\end{abstract}

Keywords: Newton's method, Aggregation function, Order of convergence.

\section{INTRODUCTION}

Having in mind that Newton's methods have a wide range of applications, a new modification of Newton's method is presented. Proposed method for solving nonlinear equations is based on aggregation function. Some existing well-known modifications of Newton's method, based on arithmetic, geometric, harmonic and root-power mean are very closely related to the special members of this method. The convergence properties of the proposed method are discussed and it is shown that the order of convergence for multiple roots is one. It is proved that the iterative sequence defined by this method converges to roots, for any initial iteration sufficiently close to root.

The rest of the paper is as follows. In Section 2 we briefly describe the concepts of aggregation functions. Definition of the method and analysis of its convergence are presented in Section 3. Finally, sections 4 we show the computational results of some relevant numerical tests to compare the efficiencies of the proposed aggregation methods (including Newton's method).

\section{AN AGGREGATION FUNCTIONS}

In this section we present a brief recall of definitions, examples and properties of aggregation functions. Let $I$ be interval real number.

Definition 1. An $n$-ary aggregation function is a function $\mathcal{A}_{[n]}$ : $I^{n} \rightarrow I$ such that

i) $\mathcal{A}_{[n]}\left(x_{1}, \ldots, x_{n}\right) \leq \mathcal{A}_{[n]}\left(y_{1}, \ldots, y_{n}\right)$ whenever $x_{i} \leq y_{i}$ for all $i \in\{1, \ldots, n\}$ ( $\mathcal{A}$ is monotonically increasing function in all its arguments). ii) $\inf _{x \in I^{n}} \mathcal{A}_{[n]}(x)=\inf I$ and $\sup _{x \in I^{n}} \mathcal{A}_{[n]}(x)=\sup I, x=\left(x_{1}, \ldots, x_{n}\right)$ (boundary condition).

An aggregation function is a function $\mathcal{A}: \bigcup_{n \in \mathbb{N}} I^{n} \rightarrow I$ such that for $n=1$ holds $\mathcal{A}(x)=x$, for all $x \in I$, whose restriction is $\left.\mathcal{A}\right|_{I^{n}}=\mathcal{A}_{[n]}$, for any $n \in \mathbb{N}$.

For aggregation function is required to comply with additional features such as

iii) $\mathcal{A}_{[n]}(x, x, \ldots, x)=x$ for all $x \in I\left(\mathcal{A}_{[n]}\right.$ is idempotent function).

iv) $\mathcal{A}_{[n]}\left(x_{1}, \ldots, x_{n}\right)=\mathcal{A}_{[n]}\left(x_{p_{1}}, \ldots, x_{p_{n}}\right)$ for any permutation $\left(p_{1}, \ldots, p_{n}\right)$ of set $\{1, \ldots, n\}\left(\mathcal{A}_{[n]}\right.$ is symmetric function in all its arguments).

v) $\mathcal{A}_{[n]}$ is continuous function.

vi) $\mathcal{A}_{[n]} \in C^{\ell}\left(\left(I^{\circ}\right)^{n}\right)$ (The function $\mathcal{A}_{[n]}$ has continuous derivatives up to the order $\ell$ in all variables).

Remark. It is customary to take the $I=[0,1]$. In this case boundary condition reduces to $\mathcal{A}(0, \ldots, 0)=0$ and $\mathcal{A}(1, \ldots, 1)=1$.

Here are some examples that we will use in our future work, in the case that is $n=2$, when the aggregation function is defined Table 1.

Root-power means

$$
M_{p}(a, b)=\left(\frac{a^{p}+b^{p}}{2}\right)^{1 / p} \quad(p \neq 0) .
$$

Marginal members of these classes are $M_{0}=G=M_{\log a}$, which is the geometric mean, while $M_{\infty}=\operatorname{Max}$ and $M_{-\infty}=$ Min. So, $A=M_{1}, Q=M_{2}$ and $C=M_{3}$.

Thus, we are going to present a definition, some examples and properties of aggregation functions (see (Grabisch et al., 2009; Klement et al., 2000; Klir \& Yuan, 1995)). 
Table 1.

\begin{tabular}{|c|c|c|}
\hline \hline $\mathcal{A}(a, b)=$ & Mean & Parameter \\
\hline \hline$\frac{a+b}{2}$ & Arithmetic $(A)$ & \\
\hline$\frac{2}{\frac{1}{a}+\frac{1}{b}}$ & Harmonic $(H)$ & \\
\hline$\sqrt{a b}$ & Geometric $(G)$ & \\
\hline$\sqrt{\frac{a^{2}+b^{2}}{2}}$ & Quadratic $(Q)$ & \\
\hline$\sqrt[3]{\frac{a^{3}+b^{3}}{2}}$ & Cubic $(C)$ & \\
\hline$\frac{a^{p} b^{1-p}+a^{1-p} b^{p}}{2}$ & Heinz $\left(Z_{p}\right)$ & $p \neq 0$ \\
\hline$\left.\frac{a^{p}+(a b)^{p / 2}+b^{p}}{3}\right)^{1 / p}$ & Generalized & \\
\hline$\frac{a^{s} b^{t}+a^{t} b^{s}}{2}$ & Heron $\left(H_{p}\right)$ & $s=\frac{1+\sqrt{p}}{2}, t=\frac{1-\sqrt{p}}{2}$ \\
\hline$\frac{a^{2}+b^{2}}{a+b}$ & Contraharmonic $(\mathrm{CH})$ & \\
\hline$\frac{a^{p}+b^{p}}{a^{p-1}+b^{p-1}}$ & Contraharmonic $(G C H)$ & \\
\hline \hline
\end{tabular}

Here we consider the aggregation functions as functions of two variables.

The methods obtained for a given aggregation function will be given the appropriate name. Thus, the quadratic and cubic mean give quadratic mean Newton's method (shortly QN method) and cubic mean Newton's method (shortly $\mathrm{CN}$ method). The contraharmonic mean, generalized contraharmonic mean, Heinz mean, Heron mean, symmetric mean, give contraharmonic mean Newton's method (shortly CHN method), generalized contraharmonic mean Newton's method (shortly GCHN method), Heinz mean Newton's method (shortly HZN method), Heron mean Newton's method (shortly HRN method) and symmetric mean Newton's method (shortly SMN method), respectively.

Definition 2. A quasi aggregation function of class $\ell$ is a function $\mathcal{A}: I^{n} \rightarrow I, n \in \mathbb{N}$ (where $I$ is the interval of real numbers) such that $\mathcal{A}$ is idempotent, symmetric and $\mathcal{A} \in C^{\ell}\left(I^{\circ}\right), \ell \in \mathbb{N}$.

Lemma 3. If $\mathcal{A}: I^{n} \rightarrow I, n \in \mathbb{N}$ is a quasi aggregation function of class $\ell$, then

i) $\mathcal{A}_{x_{i}}(x, x, \ldots, x)=\mathcal{A}_{x_{j}}(x, x, \ldots, x), x \in I, i, j=1, \ldots, n$.

ii) $\mathcal{A}_{x_{i}}(x, x, \ldots, x)=\frac{1}{n}, x \in I, i, j=1, \ldots, n$,

where $\mathcal{A}_{x_{i}}$ is $\frac{\partial \mathcal{A}}{\partial x_{i}}$.

$$
\text { For } n=2, \mathcal{A}_{x}(x, x)=\mathcal{A}_{y}(x, x)=\frac{1}{2} \text {. }
$$

Lemma 4. If $\mathcal{A}: \bigcup_{n \in \mathbb{N}} I^{n} \rightarrow I$ is a quasi aggregation function of class $\ell$, (signed $\frac{\partial \mathcal{A}}{\partial x_{i}}$ with $\mathcal{A}_{x_{i}}$ ) then holds
i) $\mathcal{A}_{x}(x, y)=\mathcal{A}_{y}(y, x)$;
ii) $\mathcal{A}_{x x}(x, y)=\mathcal{A}_{y y}(y, x)$;
iii) $\mathcal{A}_{x y}(x, y)=\mathcal{A}_{y x}(y, x)$;
iv) $\mathcal{A}_{x y}(x, y)=\mathcal{A}_{x y}(y, x)\left(A_{x y}\right.$ is symetric function $)$;
v) $\mathcal{A}_{x x y}(x, y)=A_{y y x}(y, x)$;
vi) $\mathcal{A}_{x x x}(x, y)=\mathcal{A}_{y y y}(y, x)$.

\section{DEFINITION OF THE METHOD AND ANALYSIS OF ITS CONVERGENCE}

Calculating the real root $\alpha$ of nonlinear equation

$$
f(x)=0, \quad f: I \subseteq \mathbb{R} \rightarrow \mathbb{R} .
$$

is a common problem in the field of applied mathematics.

The root $\alpha$ is said to be simple if $f(\alpha)=0$ and $f^{\prime}(\alpha) \neq 0$. If $f(\alpha)=f^{\prime}(\alpha)=\ldots=f^{(k-1)}(\alpha)=0$ and $f^{(k)}(\alpha) \neq 0$ for $k \geq 1$ then $\alpha$ is of multiplicity $k$.

If the sequence $\left\{x_{n}\right\}$ tends to a limit $\alpha$ such that

$$
\lim _{n \rightarrow \infty} \frac{x_{n+1}-\alpha}{\left(x_{n}-\alpha\right)^{q}}=C,
$$

for some $C \neq 0$ and $q \geq 1$, then the order of convergence of the sequence $\left\{x_{n}\right\}$ is $q$ and $C$ is known as the asymptotic error constant (AEC) (see (Wait, 1979)). If $q=1, q=2$ or $q=3$, the convergence is said to be linear, quadratic or cubic, respectively. Let $e_{n}=x_{n}-\alpha$ be the error in the $n^{\text {th }}$ iteration of the method which produces the sequence $\left\{x_{n}\right\}$. Then, the relation (2) can be rewritten as

$$
e_{n+1}=C e_{n}^{q}+O\left(e_{n}^{q+1}\right)=O\left(e_{n}^{q}\right)
$$

which is called the error equation. The value of $q$ is called the order of convergence and $C$ is known as the asymptotic error constant of this method.

There are several classical methods for solving the equation (1) and the most famous is Newton's method (shortly N method) defined by

$$
x_{n+1}=x_{n}-\frac{f\left(x_{n}\right)}{f^{\prime}\left(x_{n}\right)}, \quad n=0,1, \ldots,
$$

where $x_{0}$ is the initial guess that is sufficiently close to $\alpha$. Newton's method is quadratical convergence techniques for simple roots with the error equation

$$
e_{n+1}=\frac{f^{\prime \prime}(\alpha)}{2 f^{\prime}(\alpha)} \cdot e_{n}^{2}+O\left(e_{n}^{3}\right)
$$

Below are some examples of two-step Newton's methods determined with

$$
x_{n+1}=x_{n}-\frac{f\left(x_{n}\right)}{F\left(f^{\prime}\left(x_{n}\right), f^{\prime}\left(\tilde{x}_{n}\right)\right)},
$$

where $\tilde{x}_{n}=x_{n}-\frac{f\left(x_{n}\right)}{f^{\prime}\left(x_{n}\right)}, n=0,1, \ldots$

The special cases of this method, defined by (4), for simple zeros can be founded in Weerakoon and Fernando (Weerakoon \& Fernando, 2000) $(F=A)$, Özban (Özban, 2004) $(F=H)$, Lukić and Ralević (Lukić \& Ralević, 2008) ( $F=G)$, Also Ralević and Lukić in (Ralević \& Lukić, 2005),(Lukić et al., 2006) ( $\left.F=M_{p}\right)$. Certain generalizations, modifications, improvements and further analysis of the mentioned mean-based methods one can find in (Babajee \& Dauhoo, 2006; Frontini \& Sormani, 2003; Herceg \& Herceg, 2013b,a; Homeier, 2003; McDougall \& Wotherspoon, 2014). 
For reasons of feasibility of the method, the authors of (Lukić \& Ralević, 2008; Lukić et al., 2006) and (Ralević \& Lukić, 2005) take $\operatorname{sign}\left(f^{\prime}\left(x_{0}\right)\right) \cdot G\left(\left|f^{\prime}\left(x_{n}\right)\right|,\left|f^{\prime}\left(\tilde{x}_{n}\right)\right|\right)$ and $\operatorname{sign}\left(f^{\prime}\left(x_{0}\right)\right) \cdot M_{p}\left(\left|f^{\prime}\left(x_{n}\right)\right|,\left|f^{\prime}\left(\tilde{x}_{n}\right)\right|\right)$ instead of $G\left(f^{\prime}\left(x_{n}\right), f^{\prime}\left(\tilde{x}_{n}\right)\right)$ and $M_{p}\left(f^{\prime}\left(x_{n}\right), f^{\prime}\left(\tilde{x}_{n}\right)\right)$ in given methods, respectively.

The following is a generalized method based on on aggregation functions, external aggregation Newton's method will be introduced.

Let $A$ be quasi aggregation function of class $\ell$. The method which under consideration defined by

$$
\begin{aligned}
& x_{n+1}=x_{n}-\frac{f\left(x_{n}\right)}{\mathcal{A}\left(f^{\prime}\left(x_{n}\right), f^{\prime}\left(\tilde{x}_{n}\right)\right)}, \\
& \text { where } \tilde{x}_{n}=x_{n}-\frac{f\left(x_{n}\right)}{f^{\prime}\left(x_{n}\right)}, \quad n=0,1, \ldots
\end{aligned}
$$

we call external aggregation Newton's method, shortly EANM.

The next theorem (see Ralević \& Ćebić (2019)) hold.

Theorem 5. Let $f: I \subseteq \mathbb{R} \rightarrow \mathbb{R}$ where I is an open interval. Assume that $f$ is sufficiently differentiable function in the interval $I$ and $f$ has a simple root $\alpha \in I$. If $x_{0}$ is sufficiently close to $\alpha$, then the EANM converges cubically and satisfies the following error equation:

$$
e_{n+1}=e_{n}^{3}\left(c_{2}^{2}\left(2 f^{\prime}(\alpha) \mathcal{A}_{x x}\left(f^{\prime}(\alpha), f^{\prime}(\alpha)\right)+1\right)+\frac{1}{2} c_{3}\right)+o\left(e_{n}^{3}\right)
$$

where $e_{n}=x_{n}-\alpha, c_{j}=\frac{f^{(j)}(\alpha)}{j ! f^{\prime}(\alpha)}$ for $j=1,2,3, \ldots$ are constants, and $A$ is a generalized aggregation operator of class 3.

Example 6. If $\mathcal{A}(x, y)=M_{p}(x y)=\left(\frac{x^{p}+y^{p}}{2}\right)^{\frac{1}{p}}$, we have that the error equation is:

$$
e_{n+1}=\frac{1}{2}\left((p+1) c_{2}^{2}+c_{3}\right) e_{n}^{3}+o\left(e_{n}^{3}\right)
$$

In the following Theorem we give the error equation of EANM method in a case when we approximate multiple roots.

Theorem 7. Let $f: I \subseteq \mathbb{R} \rightarrow \mathbb{R}$, where $I$ is an open interval. Assume that $f$ is sufficiently differentiable function on the interval $I$ and $f$ has a multiple root of multiplicity $k>1$ in $\alpha \in$ I. If $x_{0}$ is sufficiently close to $\alpha$, then the method (5) converges linearly to $\alpha$ and satisfies the following error equation:

$$
e_{n+1}=\left(1-\frac{2}{k\left(1+\left(\frac{k-1}{k}\right)^{k-1}\right)}\right) e_{n}+\frac{2(K-1) d_{k+1}}{k\left(1+\left(\frac{k-1}{k}\right)^{k-1}\right)} e_{n}^{2}+o\left(e_{n}^{2}\right)
$$

where $e_{n}=x_{n}-\alpha, d_{k+i}=\frac{f^{(k+i)}(\alpha) /(k+i) !}{f^{(k)}(\alpha) / k !}$ and $K=\frac{\frac{k+1}{k}+\left(\frac{k-1}{k}\right)^{k-1} \cdot \frac{k^{2}+k-1}{k^{2}}}{1+\left(\frac{k-1}{k}\right)^{k-1}}$.

Proof. Since the function $f$ has the multiple root $\alpha$ of multiplicity $k$, i.e. $f(\alpha)=f^{\prime}(\alpha)=\ldots=f^{(k-1)}(\alpha)=0, f^{(k)}(\alpha) \neq 0$, using
Taylor expansion of $f\left(x_{n}\right)$ at $\alpha$, we obtain

$$
\begin{aligned}
f\left(x_{n}\right)= & f(\alpha)+f^{\prime}(\alpha) e_{n}+\frac{1}{2 !} f^{\prime \prime}(\alpha) e_{n}^{2}+\ldots \\
& +\frac{1}{k !} f^{(k)}(\alpha) e_{n}^{k}+\frac{1}{(k+1) !} f^{(k+1)}(\alpha) e_{n}^{k+1} \\
& +\frac{1}{(k+2) !} f^{(k+2)}(\alpha) e_{n}^{k+2}+o\left(e_{n}^{k+2}\right) \\
= & \frac{1}{k !} f^{(k)}(\alpha) e_{n}^{k}+\frac{1}{(k+1) !} f^{(k+1)}(\alpha) e_{n}^{k+1} \\
& +\frac{1}{(k+2) !} f^{(k+2)}(\alpha) e_{n}^{k+2}+o\left(e_{n}^{k+2}\right) \\
= & \frac{f^{(k)}(\alpha)}{k !}\left[e_{n}^{k}+\frac{f^{(k+1)}(\alpha)}{(k+1) f^{(k)}(\alpha)} e_{n}^{k+1}\right. \\
& \left.+\frac{f^{(k+2)}(\alpha)}{(k+2)(k+1) f^{(k)}(\alpha)} e_{n}^{k+2}+o\left(e_{n}^{k+2}\right)\right], \\
= & \frac{f^{(k)}(\alpha)}{k !}\left[e_{n}^{k}+d_{k+1} e_{n}^{k+1}+d_{k+2} e_{n}^{k+2}+o\left(e_{n}^{k+2}\right)\right] .
\end{aligned}
$$

Similarly,

$$
\begin{aligned}
& f^{\prime}\left(x_{n}\right) \\
= & f^{\prime}(\alpha)+f^{\prime \prime}(\alpha) e_{n}+\frac{f^{\prime \prime \prime}(\alpha)}{2 !} e_{n}^{2}+\ldots+\frac{f^{(k)}(\alpha)}{(k-1) !} e_{n}^{k-1} \\
& +\frac{f^{(k+1)}(\alpha)}{k !} e_{n}^{k}+\frac{f^{(k+2)}(\alpha)}{(k+1) !} e_{n}^{k+1}+o\left(e_{n}^{k+1}\right) \\
= & \frac{f^{(k)}(\alpha)}{(k-1) !} e_{n}^{k-1}+\frac{f^{(k+1)}(\alpha)}{k !} e_{n}^{k}+\frac{f^{(k+2)}(\alpha)}{(k+1) !} e_{n}^{k+1}+o\left(e_{n}^{k+1}\right) \\
= & \frac{f^{(k)}(\alpha)}{k !}\left[k e_{n}^{k-1}+(k+1) d_{k+1} e_{n}^{k}+(k+2) d_{k+2} e_{n}^{k+1}+o\left(e_{n}^{k+1}\right)\right] \\
= & \frac{f^{(k)}(\alpha)}{(k-1) !} e_{n}^{k-1}\left[1+\frac{k+1}{k} d_{k+1} e_{n}+\frac{k+2}{k} d_{k+2} e_{n}^{2}+o\left(e_{n}^{2}\right)\right]
\end{aligned}
$$

If we divide (8) by (9), we have

$$
\begin{aligned}
\frac{f\left(x_{n}\right)}{f^{\prime}\left(x_{n}\right)}= & \frac{1}{k} e_{n}\left(1+d_{k+1} e_{n}+d_{k+2} e_{n}^{2}+o\left(e_{n}^{2}\right)\right) \\
& \cdot\left(1+\frac{k+1}{k} d_{k+1} e_{n}+\frac{k+2}{k} d_{k+2} e_{n}^{2}+o\left(e_{n}^{2}\right)\right)^{-1} \\
= & \frac{e_{n}}{k}\left(1+d_{k+1} e_{n}+d_{k+2} e_{n}^{2}+o\left(e_{n}^{2}\right)\right) \cdot\left[1-\left(\frac{k+1}{k} d_{k+1} e_{n}\right.\right. \\
& \left.+\frac{k+2}{k} d_{k+2} e_{n}^{2}+o\left(e_{n}^{2}\right)\right)+ \\
& \left.\left(\frac{k+1}{k} d_{k+1} e_{n}+\frac{k+2}{k} d_{k+2} e_{n}^{2}+o\left(e_{n}^{2}\right)\right)^{2}+o\left(e_{n}^{2}\right)\right] \\
= & \frac{e_{n}}{k}\left(1+d_{k+1} e_{n}+d_{k+2} e_{n}^{2}+o\left(e_{n}^{2}\right)\right) \cdot\left[1-\frac{k+1}{k} d_{k+1} e_{n}\right. \\
& \left.+\left(\left(\frac{k+1}{k}\right)^{2} d_{k+1}^{2}-\frac{k+2}{k} d_{k+2}\right) e_{n}^{2}+o\left(e_{n}^{2}\right)\right] \\
= & \frac{e_{n}}{k}\left[1-\frac{1}{k} d_{k+1} e_{n}+\left(\frac{k+1}{k^{2}} d_{k+1}^{2}-\frac{2}{k} d_{k+2}\right) e_{n}^{2}+o\left(e_{n}^{2}\right)\right] .
\end{aligned}
$$

From $\tilde{x}_{n}=x_{n}-\frac{f\left(x_{n}\right)}{f^{\prime}\left(x_{n}\right)}$, we get

$$
\begin{aligned}
& \tilde{x}_{n}-\alpha=e_{n}-\frac{f\left(x_{n}\right)}{f^{\prime}\left(x_{n}\right)} \\
& =\frac{k-1}{k} e_{n}+\frac{1}{k^{2}} d_{k+1} e_{n}^{2}+\frac{1}{k^{3}}\left(2 k d_{k+2}-(k+1) d_{k+1}^{2}\right) e_{n}^{3}+o\left(e_{n}^{3}\right) \\
& =\frac{k-1}{k} e_{n}\left(1+\frac{1}{k(k-1)} d_{k+1} e_{n}+\frac{1}{k^{2}(k-1)}\left(2 k d_{k+2}-(k+1) d_{k+1}^{2}\right) e_{n}^{2}+o\left(e_{n}^{2}\right)\right),
\end{aligned}
$$

and

$$
\begin{aligned}
& f^{\prime}\left(\tilde{x}_{n}\right) \\
= & f^{\prime}(\alpha)+f^{\prime \prime}(\alpha)\left(\tilde{x}_{n}-\alpha\right)+\frac{1}{2 !} f^{\prime \prime \prime}(\alpha)\left(\tilde{x}_{n}-\alpha\right)^{2}+\ldots \\
& +\frac{1}{(k-1) !} f^{(k)}(\alpha)\left(\tilde{x}_{n}-\alpha\right)^{k-1}+\frac{1}{k !} f^{(k+1)}(\alpha)\left(\tilde{x}_{n}-\alpha\right)^{k} \\
& +\frac{1}{(k+1) !} f^{(k+2)}(\alpha)\left(\tilde{x}_{n}-\alpha\right)^{k+1}+o\left(\left(\tilde{x}_{n}-\alpha\right)^{k+1}\right)
\end{aligned}
$$


$=\frac{1}{(k-1) !} f^{(k)}(\alpha)\left(\tilde{x}_{n}-\alpha\right)^{k-1}\left[1+\frac{k+1}{k} d_{k+1}\left(\tilde{x}_{n}-\alpha\right)+\right.$

$\left.\frac{k+2}{k} d_{k+2}\left(\tilde{x}_{n}-\alpha\right)^{2}+o\left(\left(\tilde{x}_{n}-\alpha\right)^{2}\right)\right]$

$=\frac{f^{(k)}(\alpha)}{(k-1) !}\left(\frac{k-1}{k}\right)^{k-1} e_{n}^{k-1}\left(1+\frac{1}{k(k-1)} d_{k+1} e_{n}+\right.$

$\left.\frac{1}{k^{2}(k-1)}\left(2 k d_{k+2}-(k+1) d_{k+1}^{2}\right) e_{n}^{2}+o\left(e_{n}^{2}\right)\right)^{k-1}$

$\cdot\left[1+\frac{k+1}{k} d_{k+1} \cdot \frac{k-1}{k} e_{n}\left(1+\frac{1}{k(k-1)} d_{k+1} e_{n}+\right.\right.$

$\left.\frac{1}{k^{2}(k-1)}\left(2 k d_{k+2}-(k+1) d_{k+1}^{2}\right) e_{n}^{2}+o\left(e_{n}^{2}\right)\right)$

$+\frac{k+2}{k} d_{k+2} \cdot\left(\frac{k-1}{k} e_{n}\right)^{2}\left(1+\frac{1}{k(k-1)} d_{k+1} e_{n}+\frac{1}{k^{2}(k-1)}\left(2 k d_{k+2}\right.\right.$

$\left.\left.\left.-(k+1) d_{k+1}^{2}\right) e_{n}^{2}+o\left(e_{n}^{2}\right)\right)^{2}+o\left(e_{n}^{2}\right)\right]$

$=\frac{f^{(k)}(\alpha)}{(k-1) !}\left(\frac{k-1}{k}\right)^{k-1} e_{n}^{k-1}\left[1+(k-1)\left(\frac{1}{k(k-1)} d_{k+1} e_{n}+\frac{1}{k^{2}(k-1)}\right.\right.$

$\left.\left(2 k d_{k+2}-(k+1) d_{k+1}^{2}\right) e_{n}^{2}+o\left(e_{n}^{2}\right)\right)$

$\left.+\frac{(k-1)(k-2)}{2}\left(\frac{1}{k(k-1)} d_{k+1} e_{n}+o\left(e_{n}\right)\right)^{2}+o\left(e_{n}^{2}\right)\right]$

$\cdot\left[1+\frac{k+1}{k} d_{k+1} \cdot \frac{k-1}{k} e_{n}\left(1+\frac{1}{k(k-1)} d_{k+1} e_{n}+o\left(e_{n}\right)\right)\right.$

$\left.+\frac{k+2}{k} d_{k+2}\left(\frac{k-1}{k}\right)^{2} e_{n}^{2}+o\left(e_{n}^{2}\right)\right]$

$=\frac{f^{(k)}(\alpha)}{(k-1) !}\left(\frac{k-1}{k}\right)^{k-1} e_{n}^{k-1}\left[1+\frac{1}{k} d_{k+1} e_{n}+\frac{1}{k^{2}}\left(2 k d_{k+2}\right.\right.$

$\left.\left.-(k+1) d_{k+1}^{2}\right) e_{n}^{2}+\frac{(k-1)(k-2)}{2} \cdot \frac{1}{k^{2}(k-1)^{2}} d_{k+1}^{2} e_{n}^{2}+o\left(e_{n}^{2}\right)\right]$

$\cdot\left[1+\frac{k^{2}-1}{k^{2}} d_{k+1} e_{n}+\frac{k+1}{k^{3}} d_{k+1}^{2} e_{n}^{2}+\frac{(k+2)(k-1)^{2}}{k^{3}} d_{k+2} e_{n}^{2}+o\left(e_{n}^{2}\right)\right]$

$=\frac{f^{(k)}(\alpha)}{(k-1) !}\left(\frac{k-1}{k}\right)^{k-1} e_{n}^{k-1}\left[1+\frac{1}{k} d_{k+1} e_{n}+\frac{1}{k^{2}}\left(2 k d_{k+2}\right.\right.$

$\left.\left.-(k+1) d_{k+1}^{2}+\frac{k-2}{2(k-1)} d_{k+1}^{2}\right) e_{n}^{2}+o\left(e_{n}^{2}\right)\right]$

$\cdot\left[1+\frac{k^{2}-1}{k^{2}} d_{k+1} e_{n}+\frac{1}{k^{3}}\left((k+2)(k-1)^{2} d_{k+2}\right.\right.$

$\left.\left.+(k+1) d_{k+1}^{2}\right) e_{n}^{2}+o\left(e_{n}^{2}\right)\right]$

$=\frac{f^{(k)}(\alpha)}{(k-1) !}\left(\frac{k-1}{k}\right)^{k-1} e_{n}^{k-1}\left[1+\frac{k^{2}+k-1}{k^{2}} d_{k+1} e_{n}+\right.$

$\left(\frac{k^{3}+2 k^{2}-3 k+2}{k^{3}} d_{k+2}+\frac{k-2}{2 k^{2}(k-1)} d_{k+1}^{2}\right) e_{n}^{2}+o\left(e_{n}^{2}\right)$

By lema , the properties $\mathcal{A}_{x}(\alpha, \alpha)=\mathcal{A}_{y}(\alpha, \alpha)=\frac{1}{2}$ holds, and by idempotency of $\mathcal{A}$ (i.e. $\mathcal{A}(\alpha, \alpha)=\alpha$ ), and taking $a=b=$ $\frac{f^{(k)}(\alpha)}{(k-1) !} e_{n}^{k-1}$, and $x=f^{\prime}\left(x_{n}\right), y=f^{\prime}\left(\tilde{x}_{n}\right)$, in Taylor's expansion

$$
\begin{aligned}
\mathcal{A}(x, y)=\mathcal{A}(a, b)+ & \frac{1}{1 !}\left[\mathcal{A}_{x}(a, b)(x-a)+\mathcal{A}_{y}(a, b)(y-b)\right] \\
& +o(\|(x-a, y-b)\|),
\end{aligned}
$$

follows

$$
\mathcal{A}\left(f^{\prime}\left(x_{n}\right), f^{\prime}\left(\tilde{x}_{n}\right)\right)=\frac{f^{(k)}(\alpha)}{(k-1) !} e_{n}^{k-1}+\frac{1}{2}\left[\frac { f ^ { ( k ) } ( \alpha ) } { ( k - 1 ) ! } e _ { n } ^ { k - 1 } \left[\frac{k+1}{k} d_{k+1} e_{n}+\right.\right.
$$
$\left.\frac{k+2}{k} d_{k+2} e_{n}^{2}+o\left(e_{n}^{2}\right)\right]+\left(\frac{f^{(k)}(\alpha)}{(k-1) !}\left(\frac{k-1}{k}\right)^{k-1} e_{n}^{k-1}\left[1+\frac{k^{2}+k-1}{k^{2}} d_{k+1} e_{n}+o\left(e_{n}\right)\right]-\right.$ $\left.\left.\frac{f^{(k)}(\alpha)}{(k-1) !} e_{n}^{k-1}\right)\right]+o\left(e_{n}^{k}\right)=\frac{1}{2} \frac{f^{(k)}(\alpha)}{(k-1) !} e_{n}^{k-1}\left[1+\left(\frac{k-1}{k}\right)^{k-1}+\left(\frac{k+1}{k}+\left(\frac{k-1}{k}\right)^{k-1}\right.\right.$. $\left.\left.\frac{k^{2}+k-1}{k^{2}}\right) d_{k+1} e_{n}+o\left(e_{n}\right)\right]$.

Now, the formula (5) is transformed into

$$
\begin{aligned}
& x_{n+1}-\alpha=x_{n}-\alpha-\frac{f\left(x_{n}\right)}{A\left(f^{\prime}\left(x_{n}\right), f^{\prime}\left(\tilde{x}_{n}\right)\right)}, \text { i.e., } \\
& e_{n+1}= e_{n}-\frac{f\left(x_{n}\right)}{\mathcal{A}\left(f^{\prime}\left(x_{n}\right), f^{\prime}\left(\tilde{x}_{n}\right)\right)} \\
&= e_{n}-\frac{\frac{f^{(k)}(\alpha)}{k !} e_{n}^{k}\left[1+d_{k+1} e_{n}+d_{k+2} e_{n}^{2}+o\left(e_{n}^{2}\right)\right]}{\frac{1}{2} \frac{f^{(k)}(\alpha)}{(k-1) !} e_{n}^{k-1}\left[1+\left(\frac{k-1}{k}\right)^{k-1}+\left(\frac{k+1}{k}+\left(\frac{k-1}{k}\right)^{k-1} \cdot \frac{k^{2}+k-1}{k^{2}}\right) d_{k+1} e_{n}+o\left(e_{n}\right)\right]} \\
&= e_{n}-\frac{2}{k\left(1+\left(\frac{k-1}{k}\right)^{k-1}\right)} e_{n}\left[1+d_{k+1} e_{n}+d_{k+2} e_{n}^{2}+o\left(e_{n}^{2}\right)\right] \\
& \cdot\left[1+K d_{k+1} e_{n}+o\left(e_{n}\right)\right]^{-1} \\
&= e_{n}-\frac{2}{k\left(1+\left(\frac{k-1}{k}\right)^{k-1}\right)} e_{n}\left[1+d_{k+1} e_{n}+d_{k+2} e_{n}^{2}+o\left(e_{n}^{2}\right)\right] \\
& \cdot\left[1-K d_{k+1} e_{n}+o\left(e_{n}\right)\right] \\
&= e_{n}-\frac{2}{k\left(1+\left(\frac{k-1}{k}\right)^{k-1}\right)} e_{n}\left[1+\left(d_{k+1}-K d_{k+1}\right) e_{n}+o\left(e_{n}\right)\right] \\
&=\left(1-\frac{2}{k\left(1+\left(\frac{k-1}{k}\right)^{k-1}\right)}\right) e_{n}+\frac{2}{k\left(1+\left(\frac{k-1}{k}\right)^{k-1}\right)}(K-1) d_{k+1} e_{n}^{2}+o\left(e_{n}^{2}\right)
\end{aligned}
$$

where is $K=\frac{\frac{k+1}{k}+\left(\frac{k-1}{k}\right)^{k-1} \cdot \frac{k^{2}+k-1}{k^{2}}}{1+\left(\frac{k-1}{k}\right)^{k-1}}$.

Remark. In the proofs of the theorems we used the known properties of the function $o$ :

i) $o(f)+o(f)=o(f)$, ii) $o(o(f))=o(f)$, iii) $f_{1} \cdot o\left(f_{2}\right)=o\left(f_{1} f_{2}\right)$,

iv) $o(c f)=o(f),(c-$ const. $) v) o(f+o(f))=o(f)$.

Recall some notions from functional analysis (see Rudin (1991)).

If $f$ is a mapping of the set of $X$ to itself, then the point $x \in X$ is called a fixed (stationary) point of map $f$ if $f(x)=x$.

We say that a map $f: X \rightarrow Y$ of the metric space $\left(X, d_{1}\right)$ in the metric space $\left(Y, d_{2}\right)$, is a contraction if there exists a real number $\lambda \in(0,1)$ such that for every $x_{1}, x_{2} \in X$

$$
d_{2}\left(f\left(x_{1}\right), f\left(x_{2}\right)\right) \leq \lambda d_{1}\left(x_{1}, x_{2}\right) .
$$

The number $\lambda$ is called the coefficient of contraction, and $f$ a contraction mapping.

Banach fixed point theorem Rudin (1991):

Theorem 8. If $(X, d)$ is a complete metric space and $f: X \rightarrow X a$ contraction with coefficient $\lambda$, then there is one and only one fixed point $x \in X$ of the mapping $f$.

Consider the function $\varphi(x)=x-\frac{f(x)}{g(x)}$, where $g(x)=$ $\mathcal{A}\left(f^{\prime}(x), f^{\prime}\left(x-\frac{f(x)}{f^{\prime}(x)}\right)\right)$. Then EANM method can be written as

$$
x_{n+1}=\varphi\left(x_{n}\right) .
$$

Theorem 9. Let $f$ be a real valued and $f \in C^{3}(I)$ on the closed interval I, $\alpha$ a simple root of $f$ belonging to the interior of $I$, then the function $\varphi$ a contraction in some neighbourhood of $\alpha$ where the function value is assumed to be small enough.

Proof. It is easy to see that

$$
\varphi^{\prime}(x)=1-\frac{f^{\prime}(x)}{g(x)}+\frac{f(x) g^{\prime}(x)}{(g(x))^{2}} .
$$

If we denote $\frac{\partial \mathcal{A}}{\partial a}(a, b)$ as $\mathcal{A}_{a}(a, b)$ and $\frac{\partial \mathcal{A}}{\partial b}(a, b)$ as $\mathcal{A}_{b}(a, b)$, then

$$
\begin{gathered}
g^{\prime}(x)=\mathcal{A}_{a}\left(f^{\prime}(x), f^{\prime}\left(x-\frac{f(x)}{f^{\prime}(x)}\right)\right) \cdot \frac{\partial a}{\partial x}+\mathcal{A}_{b}\left(f^{\prime}(x), f^{\prime}\left(x-\frac{f(x)}{f^{\prime}(x)}\right)\right) \cdot \frac{\partial b}{\partial x} \\
=\mathcal{A}_{a}\left(f^{\prime}(x), f^{\prime}\left(x-\frac{f(x)}{f^{\prime}(x)}\right) \cdot f^{\prime \prime}(x)\right. \\
+\mathcal{A}_{b}\left(f^{\prime}(x), f^{\prime}\left(x-\frac{f(x)}{f^{\prime}(x)}\right)\right) \cdot f^{\prime \prime}\left(x-\frac{f(x)}{f^{\prime}(x)}\right) \cdot \frac{f(x) f^{\prime \prime}(x)}{\left(f^{\prime}(x)\right)^{2}} .
\end{gathered}
$$

Because $f(\alpha)=0$ and idempotency of $\mathcal{A}$ follows $g(\alpha)=$ $\mathcal{A}\left(f^{\prime}(\alpha), f^{\prime}(\alpha)\right)=f^{\prime}(\alpha)$ and by lema, $g^{\prime}(\alpha)=\mathcal{A}_{a}\left(f^{\prime}(\alpha), f^{\prime}(\alpha)\right)$. $\left.f^{\prime \prime}(\alpha)+\mathcal{A}_{b}\left(f^{\prime}(\alpha), f^{\prime}(\alpha)\right) \cdot f^{\prime \prime}(\alpha)\right) \cdot 0=\frac{f^{\prime \prime}(\alpha)}{2}$.

Using Taylor expansion of $F_{1}(x)=\frac{f^{\prime}(x)}{g(x)}$ at $\alpha$, we obtain

$$
\begin{aligned}
F_{1}(x) & =F_{1}(\alpha)+F_{1}^{\prime}(\alpha)(x-\alpha)+O\left((x-\alpha)^{2}\right) \\
& =\frac{f^{\prime}(\alpha)}{g(\alpha)}+\frac{g^{\prime}(\alpha) f^{\prime}(\alpha)-g(\alpha) f^{\prime \prime}(\alpha)}{\left(f^{\prime}(\alpha)\right)^{2}}(x-\alpha)+O\left((x-\alpha)^{2}\right) \\
& =1-\frac{f^{\prime \prime}(\alpha)}{2 f^{\prime}(\alpha)}(x-\alpha)+O\left((x-\alpha)^{2}\right)
\end{aligned}
$$


Similarly, using Taylor expansion of $F_{2}(x)=\frac{f(x) g^{\prime}(x)}{(g(x))^{2}}$ at $\alpha$, we obtain

$$
\begin{aligned}
& F_{2}(x)=F_{2}(\alpha)+F_{2}^{\prime}(\alpha)(x-\alpha)+O\left((x-\alpha)^{2}\right)=\frac{f(\alpha) g^{\prime}(\alpha)}{(g(\alpha))^{2}}+ \\
& \frac{\left(f^{\prime}(\alpha) g^{\prime}(\alpha)+f(\alpha) g^{\prime \prime}(\alpha)\right) \cdot g^{2}(\alpha)-f(\alpha) g^{\prime}(\alpha) \cdot 2 g(\alpha)\left(g^{\prime}(\alpha)\right)^{2}}{g^{4}(\alpha)} \\
& \cdot(x-\alpha)+O\left((x-\alpha)^{2}\right)=\frac{f^{\prime \prime}(\alpha)}{2 f^{\prime}(\alpha)}(x-\alpha)+O\left((x-\alpha)^{2}\right) .
\end{aligned}
$$

Now,

$$
\begin{aligned}
\left|\varphi^{\prime}(x)\right|= & \left|1-F_{1}(x)+F_{2}(x)\right| \\
= & \mid 1-1+\frac{f^{\prime \prime}(\alpha)}{2 f^{\prime}(\alpha)}+O\left((x-\alpha)^{2}\right) \\
& +\frac{f^{\prime \prime}(\alpha)}{2 f^{\prime}(\alpha)}+O\left((x-\alpha)^{2}\right) \mid \\
= & \left|\frac{f^{\prime \prime}(\alpha)}{f^{\prime}(\alpha)}(x-\alpha)+O\left((x-\alpha)^{2}\right)\right| \\
\leq & \left|\frac{f^{\prime \prime}(\alpha)}{f^{\prime}(\alpha)}\right| \cdot|x-\alpha|+\left|O\left((x-\alpha)^{2}\right)\right|
\end{aligned}
$$

If we use the definition of the function $O$, we have that there exists a positive real number $M$ and a positive real number $\delta$ such that for all $x$ from the neighbourhood $(\alpha-\delta, \alpha+\delta)$ of point $\alpha$

$$
O\left((x-\alpha)^{2}\right) \leq M|x-\alpha|^{2},
$$

is satisfied.

If we introduce the label $L=\frac{f^{\prime \prime}(\alpha)}{f^{\prime}(\alpha)}$, it follows

$$
\begin{aligned}
\left|\varphi^{\prime}(x)\right| & \leq(L+M \cdot|x-\alpha|)|x-\alpha| \\
& \leq(L+M \delta)|x-\alpha|
\end{aligned}
$$

If we choose the neighbourhood $(\alpha-\varepsilon, \alpha+\varepsilon)$ of the point $\alpha$ such that

$$
\varepsilon=\min \left\{\delta, \frac{1}{2(L+M \delta)}\right\},
$$

we get that $\left|\varphi^{\prime}(x)\right|<\frac{1}{2}$, i.e., $\varphi$ is a contraction.

For arbitrary $x \in[\alpha-\varepsilon, \alpha-\varepsilon]=I$ we have

$$
|\varphi(x)-\alpha|=|\varphi(x)-\varphi(\alpha)|=\left|\varphi^{\prime}(\bar{x})\right| \cdot|x-\alpha|<|x-\alpha|<\varepsilon,
$$

i.e., $\varphi(x) \in[\alpha-\varepsilon, \alpha-\varepsilon]$ and $\varphi: I \rightarrow I$.

Conditions of the Banach fixed point theorem are fulfilled. Thus, the mapping $\varphi$ has a unique fixed point in thats neighbourhood, and therefore the same follows for the function $f$, then EANM method ensures the sequence $\left\{x_{n}\right\}$ defined by (10) converges to $\alpha$ for any initial iteration $x_{0}$ sufficiently close to $\alpha$.

\section{NUMERICAL RESULTS AND CONCLUSIONS}

In Tables 1-5 we show the computational results of some relevant numerical tests derived from the real-life applications, to compare the efficiencies of the proposed aggregation methods (including Newton's method) for simple and multiple roots. All the tables display the number of iterations $N$ and the number of functional evaluations $N_{f}$, required to satisfy the stopping criterion $\left|x_{n+1}-x_{n}\right|+\left|f\left(x_{n+1}\right)\right|<10^{-7}$ where the maximum number of iterations is 500. Moreover, the computational order of convergence
COC given in (Weerakoon \& Fernando, 2000) has been calculated with following formula

$$
\frac{\ln \left|\left(x_{n+1}-\alpha\right) /\left(x_{n}-\alpha\right)\right|}{\ln \left|\left(x_{n}-\alpha\right) /\left(x_{n-1}-\alpha\right)\right|}
$$

\section{Test functions:}

$$
\begin{aligned}
& f_{1}(x)=e^{-x}-1-x / 5 \\
& f_{2}(x)=2 e^{-\sqrt{x}}(\sqrt{x}+1)-2 e^{-\sqrt{x+1}}\left(\sqrt{x+1}-e^{-1}\right) \\
& f_{3}(x)=x-0.5 \cos x+\pi / 4 \\
& f_{4}(x)=x^{4}+4 x^{3}-24 x^{2}+16 x+16 \\
& f_{5}(x)=x^{4}+11.5 x^{3}+47.49 x^{2}+83.06325 x+51.23266875
\end{aligned}
$$

All test examples are the real-life problems. The first function has been derived from the Planck's radiation law (see Jain (2013)), the second is the problem of minimum insurance premium determination, while the third describes the trajectory of the electron in the air gap between two parallel plates (see Maroju et al. (2017)). Here we are interested in finding simple roots $4.965114 \ldots, 0.541919808 \ldots$ and $-0.3090932 \ldots$ respectively. In the fourth and the fifth example, we are searching for multiple roots -2.85 and 2 respectively. The fourth test function arised from the beam positioning problem (see Zachary (2012)), and fifth problem is also known as a continuous stirred tank reactor problem (see Douglas (1972)).

Table 2. Numerical results for $f_{1}(x)$ and $x_{0}=3$.

\begin{tabular}{llll}
\hline method & $N$ & $N_{f}$ & COC \\
\hline $\mathrm{N}$ & 4 & 8 & 1.9504 \\
$\mathrm{AN}$ & 4 & 12 & 2.9970 \\
$\mathrm{HN}$ & 4 & 12 & 2.9944 \\
$\mathrm{GN}$ & 4 & 12 & 2.9958 \\
$\mathrm{QN}$ & 4 & 12 & 2.9981 \\
$\mathrm{CN}$ & 3 & 9 & 3.1845 \\
$\mathrm{CHN}$ & 3 & 9 & 3.1791 \\
$\mathrm{HZN}(p=1 / 4)$ & 4 & 12 & 2.9961 \\
$\mathrm{HRN}$ & 4 & 12 & 2.9966 \\
$\mathrm{SMN}(p=9)$ & 4 & 12 & 2.9999 \\
$\mathrm{GCHN}(p=3)$ & 3 & 9 & 2.7593 \\
\hline
\end{tabular}

Table 3. Numerical results for $f_{2}(x)$ and $x_{0}=0.25$.

\begin{tabular}{llll}
\hline method & $N$ & $N_{f}$ & COC \\
\hline N & 5 & 10 & 1.9998 \\
AN & 4 & 12 & 2.9979 \\
HN & 3 & 9 & 2.9479 \\
GN & 4 & 12 & 2.9988 \\
QN & - & - & - \\
CN & - & - & - \\
CHN & 4 & 12 & 2.9959 \\
HZN $(p=1 / 4)$ & 4 & 12 & 2.9986 \\
HRN & - & - & - \\
SMN $(p=9)$ & 4 & 12 & 2.9882 \\
GCHN $(p=3)$ & 4 & 12 & 2.9937 \\
\hline
\end{tabular}


Table 4. Numerical results for $f_{3}(x)$ and $x_{0}=-1.4$.

\begin{tabular}{llll}
\hline method & $N$ & $N_{f}$ & COC \\
\hline N & 5 & 10 & 1.9963 \\
AN & 4 & 12 & 3.0424 \\
HN & 4 & 12 & 3.0082 \\
GN & 4 & 12 & 3.0232 \\
QN & 4 & 12 & 3.0628 \\
CN & 4 & 12 & 3.0816 \\
CHN & 4 & 12 & 3.0856 \\
HZN $(\mathrm{p}=1 / 4)$ & 4 & 12 & 3.0277 \\
HRN & 4 & 12 & 3.0357 \\
SMN $(\mathrm{p}=9)$ & 5 & 15 & 3.0109 \\
GCHN $(\mathrm{p}=3)$ & 5 & 15 & 3.0013 \\
\hline
\end{tabular}

Table 5. Numerical results for $f_{4}(x)$ and $x_{0}=1.7$.

\begin{tabular}{llll}
\hline method & $N$ & $N_{f}$ & COC \\
\hline $\mathrm{N}$ & 22 & 44 & 1.0000 \\
$\mathrm{AN}$ & 15 & 45 & 1.0000 \\
$\mathrm{HN}$ & 12 & 36 & 1.0000 \\
$\mathrm{GN}$ & 13 & 39 & 1.0000 \\
$\mathrm{QN}$ & 12 & 36 & 3.0006 \\
$\mathrm{CN}$ & 27 & 81 & 1.0000 \\
$\mathrm{CHN}$ & 17 & 51 & 1.0000 \\
$\mathrm{HZN}(\mathrm{p}=1 / 4)$ & 14 & 42 & 1.0000 \\
$\mathrm{HRN}$ & 15 & 45 & 1.0000 \\
$\mathrm{SMN}(\mathrm{p}=9)$ & 25 & 75 & 1.0000 \\
GCHN $(\mathrm{p}=3)$ & 19 & 57 & 1.0000 \\
\hline
\end{tabular}

Table 6. Numerical results for $f_{5}(x)$ and $x_{0}=-3$.

\begin{tabular}{llll}
\hline method & $N$ & $N_{f}$ & COC \\
\hline N & 21 & 42 & 1.0000 \\
AN & 14 & 42 & 1.0000 \\
$\mathrm{HN}$ & 12 & 36 & 1.0000 \\
$\mathrm{GN}$ & 13 & 39 & 1.0000 \\
$\mathrm{QN}$ & 15 & 45 & 1.0000 \\
$\mathrm{CN}$ & 16 & 48 & 1.0000 \\
$\mathrm{CHN}$ & 16 & 48 & 1.0000 \\
$\mathrm{HZN}(\mathrm{p}=1 / 4)$ & 13 & 39 & 1.0000 \\
$\mathrm{HRN}$ & 14 & 42 & 1.0000 \\
SMN $(\mathrm{p}=9)$ & 24 & 72 & 1.0000 \\
GCHN (p=3) & 18 & 54 & 1.0000
\end{tabular}

All computational results have been carried out by the Mathematica programming package. The failure of the method is denoted by -.

All calculated COC numbers for each member of the aggregation family of methods are very close to 3 for simple roots, and very close to 1 for multiple roots, which means that those numerical results clearly confirm the theoretical analysis from previous sections. In general, it is impossible to conclude which choice of the aggregation function is the best, although it seems that for this set of test functions the harmonic mean aggregation method shows slightly better numerical results for multiple roots.

\section{ACKNOWLEDGMENT}

The author acknowledge the financial support of the Ministry of Education, Science and Technological Development of the Republic of Serbia, within the Projects No. ON 174009.

\section{REFERENCES}

Babajee, D. K. R. \& Dauhoo, M. Z. 2006. An analysis of the properties of the variants of Newton's method with third order convergence, Applied Mathematics and Computation, 183(1), pp. 659-684. doi:10.1016/j.amc.2006.05.116.

Douglas, J. M. 1972. Process Dynamics and Control: Control System Synthesis, Prentice Hall, 2. doi:10.1002/aic.690190246.

Frontini, M. \& Sormani, E. 2003. Some variant of Newton's method with third order convergence, Applied Mathematics and Computation, 140(2-3), pp. 419-426, doi:10.1016/S00963003(02)00238-2.

Grabisch, M., Marichal, J. L., Mesiar, R., \& Pap, E. 2009. Aggregation Function, Cambridge University Press.

Herceg, D. \& Herceg, D. 2013a. Means based modifications of Newton's method for solving nonlinear equations, Applied Mathematics and Computation, 219(11), pp. 6126-6133.

Herceg, D. \& Herceg, D. 2013b. Third-order modifications of Newton's method based on Stolarsky and Gini means, Journal of Computational and Applied Mathematics, 245, pp. 53-61. doi:10.1016/j.cam.2012.12.008.

Homeier, H. H. H. 2003. A modified Newton method for rootfinding with cubic convergence, J. Comput. Appl. Math., 157(1), pp. 53-61. doi:10.1016/S0377-0427(03)00391-1.

Jain, D. 2013. Families of Newton-like methods with fourthorder convergence, International Journal of computer mathematics, 90(5), pp. 1072-1082. doi:10.1080/00207160.2012.746677.

Klement, E. P., Mesiar, R., \& Pap, E. 2000. Triangular Norms, Kluwer Academic Publishers, Dordrecht. doi:10.1007/978-94015-950-7.

Klir, G. J. \& Yuan, B. 1995. Fuzzy sets and fuzzy logic, Theory and applications, Prentice Hall, New Jersey.

Lukić, T. \& Ralević, N. M. 2008. Geometric Mean Newton's Method for Simple and Multiple Roots, Applied Mathematics Letters, 21, pp. 30-36. doi:10.1016/j.aml.2007.02.010.

Lukić, T., Ralević, N. M., \& Lukity, A. 2006. Application of Aggregation Operators in Solution of Nonlinear Equations, 4th Serbian-Hungarian Joint Symposium on Intelligent Systems, September 29-30, 2006, Subotica, Serbia and Montenegro, pp. 329-339.

Maroju, P., Behl, R., \& Motsa, S. S. 2017. Some novel and optimal families of King's method with eighth and sixteenth-order of convergence, Journal of Computational and Applied Mathematics, 318, pp. 136-148. doi:10.1016/j.cam.2016.11.018.

McDougall, T. J. \& Wotherspoon, S. J. 2014. A simple modification of Newton's method to achieve convergence of order $1+2^{(1 / 2)}$, Applied Mathematics Letters, 29, pp. 20-25. doi:10.1016/j.aml.2013.10.008. 
Özban, A. Y. 2004. Some new variants of Newton's method, Appl. Math. Lett., 17, pp. 677-682. doi:10.1016/S08939659(04)90104-8.

Ralević, N. M. \& Ćebić, D. 2019. The Newton Method for Solving Nonlinear Equations Based on Aggregation operator, SYM-OPIS 2019, XLVI International Symposium on Operational Research, Kladovo, September 15-18, 2019, pp. 367-372.

Ralević, N. M. \& Lukić, T. 2005. Modification of Newton's Method Based on Root-Power Mean, Applied Linear Algebra 2005, October 13-15, Palic, Serbia and Montenegro.

Rudin, W. 1991. Functional analysis (McGraw-Hill) Wait, R.
1979. The Numerical Solution of Algebraic Equations, John Wiley Sons.

Wait, R. 1979. The Numerical Solution of Algebraic Equations. John Wiley \& Sons.

Weerakoon, S. \& Fernando, T. G. I. 2000. A variant of Newton's method with accelarated third-order convergence, Appl. Math. Lett., 13(8), pp. 87-93. doi:10.1016/S0893-9659(00)00100-2.

Zachary, J. L. 2012. Introduction to scientific programming: computational problem solving using Maple and C, Springer-Verlag New York, doi:10.1007/978-1-4612-2366-5. 